\title{
Segmentation of 3D Brain Structures in MRI Images
}

\author{
P. Narendran ${ }^{1}$ and V. Thiagarasu ${ }^{2}$ \\ ${ }^{1}$ Head \& Associate Professor, Department of Computer Science \& Research Scholar (Part Time), \\ Gobi Arts \& Science College (Autonomous), Erode, Tamil Nadu, India \\ ${ }^{2}$ Principal, Gobi Arts \& Science College (Autonomous), Erode, Tamil Nadu, India \\ E-Mail: narendranp@gmail.com, profdravt@gmail.com
}

\begin{abstract}
The main topic of this paper is to segment brain tumors, their components (edema and necrosis) and internal structures of the brain in 3D MR images. For tumor segmentation, we propose a framework that is a combination of region-based and boundary-based paradigms. In this framework, we first segment the brain using a method adapted for pathological cases and extract some global information on the tumor by symmetry based histogram analysis. The second step segments the tumor and its components. For this, we propose a new and original method that combines region and boundary information in two phases: initialization and refinement. For initialization, which is mostly region-based, we present two new methods. The first one is a new fuzzy classification method which combines the membership, typicality and neighborhood information of the voxels. The second one relies on symmetry-based histogram analysis. The initial segmentation of the tumor is refined relying on boundary information of the image. This method is a deformable model constrained by spatial relations. The spatial relations are obtained based on the initial segmentation and surrounded tissues of the tumor. The proposed method can be used for a large class of tumors in any modality of MR images. To segment a tumor and its components automatically the proposed framework needs only a contrast enhanced T1weighted image and a FLAIR image. In the case of a contrast enhanced T1-weighted image only, some user interaction will be needed. To refine the initial segmentation, we use a deformable model which is again constrained by the fused spatial relations of the structure. This method was also evaluated on 10 contrast enhanced T1-weighted images to segment the ventricles, caudate nucleus and thalamus.
\end{abstract}

Keywords: 3D, Brain, Tumor, Segmentation, MRI, Image Registration and Brain Structure

\section{INTRODUCTION}

Tumor is one of the most common brain diseases, so its diagnosis and treatment has a vital importance for more than 400000 persons per year in the world (based on the World Health Organization (WHO) estimates). On the other hand, in recent years, developments in medical imaging techniques allow us to use them in several domains of medicine, for example, computer aided pathologies diagnosis, follow-up of these pathologies, surgical planning, surgical guidance, statistical and time series (longitudinal) analysis. Among all the medical image modalities, Magnetic Resonance Imaging (MRI) is the most frequently used imaging technique in neuroscience and neurosurgery for these applications. MRI creates a 3D image which perfectly visualizes anatomic structures of the brain such as deep structures and tissues of the brain, as well as the pathologies.

Segmentation of objects, mainly anatomical structures and pathologies from MR images is a fundamental task, since the results often become the basis for other applications. Methods for performing segmentation vary widely depending on the specific application and image modality. Moreover, the segmentation of medical images is a challenging task, because they usually involve a large amount of data, they have sometimes some artifacts due to patient's motion or limited acquisition time and soft tissue boundaries are usually not well defined. The accurate segmentation of internal structures of the brain is of great interest for the study and the treatment of tumors. It aims at reducing the mortality and improving the surgical or radiotherapeutic management of tumors. In brain oncology, it is also desirable to have a descriptive human brain model that can integrate tumor information extracted from MRI data such as its localization, its type, its shape, its anatomofunctional positioning, as well as its influence on other brain structures.

Despite numerous efforts and promising results in the medical imaging community, accurate and reproducible segmentation and characterization of abnormalities are still a challenging and difficult task. Existing methods leave significant room for increased automation, applicability and accuracy.

\section{OBJECTIVES OF THE STUDY}

The first aim of this work is to develop a framework for a robust and accurate segmentation of a large class of brain tumors in MR images. Most existing methods are regionbased. They have several advantages, but line and edge information in computer vision systems are also important. The proposed method tries to combine region and edge information, thus taking advantage of both approaches while cancelling their drawbacks. 3D contrast enhanced T1weighted and FLAIR images are the inputs to perform an automatic segmentation of the solid part of tumor and the potential associated edema and necrosis [3].

We first segment the brain to remove non-brain data. However, in pathological cases, standard segmentation methods fail, in particular when the tumor is located very 
close to the brain surface. Therefore we propose an improved segmentation method, relying on the approximate symmetry plane. Then we develop two new and original methods to detect and initially segment brain tumors. The first one is a fuzzy classification method which combines membership, typicality and neighborhood information [1]. The second one relies on a symmetry-based histogram analysis. The approximate sagittal symmetry plane is first computed, and the tumor is then extracted by comparing the histograms of the two cerebral hemispheres. To refine the initial segmentation, which is not accurate enough, we use edge information. A deformable model constrained by spatial relations is applied for this purpose [8].

Segmentation of internal structures of the pathological brain is another aim of this paper. The use of prior knowledge can guide the segmentation task in medical imaging. Due to the existence of different types of tumors and consequently different effects on the brain structures, segmentation using prior knowledge such as an atlas is a difficult task. In this work we use another type of prior knowledge which reserves its properties in pathological cases.

\section{ANATOMY OF THE BRAIN}

The nervous system is commonly divided into the Central Nervous System (CNS) and the peripheral nervous system. The CNS is made up of the brain, its cranial nerves and the spinal cord. In this section we briefly study the cell structures and anatomical components of the brain. The brain consists mainly of two tissue types: Gray Matter (GM) and White Matter (WM). Gray matter is made of neuronal and glial cells, also known as neuroglia or glia that control brain activity, while the cortex is a coat of gray matter that covers the brain and the basal nuclei are the gray matter nuclei located deep within the white matter. The basal nuclei include: caudate nucleus, putamen, pallidum and claustrum. White matter fibers are myelinated axons which connect the cerebral cortex with other brain regions. The corpus callosum, a thick band of white matter fibers, connects the left and right hemispheres of the brain.

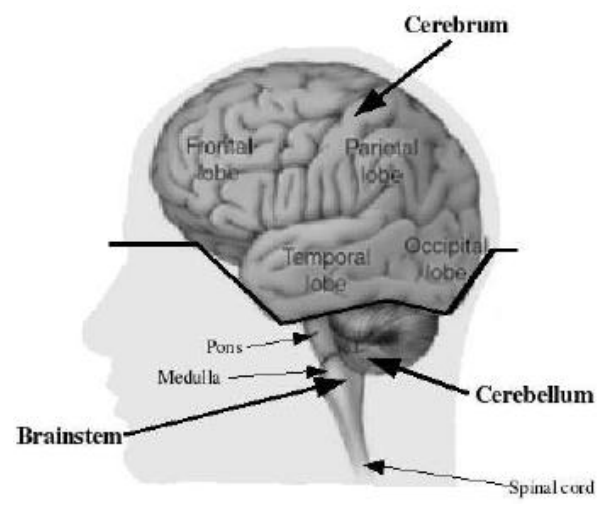

Fig. 1 Anatomy of the brain

Anatomically the brain is composed of the cerebrum, the cerebellum and the brainstem (Fig. 1). The cerebrum, which forms the major part of the brain, is divided into two major parts by the longitudinal fissure: the right and left cerebral hemispheres. Each hemisphere is divided into 4 lobes or areas: the frontal lobe in the front of the brain, the parietal lobe behind the frontal lobe, the temporal lobe on each side of the brain and the occipital lobe at the back of the brain as illustrated in Fig. 1.

The central structures of the brain, i.e. the diencephalon, include the thalamus, hypothalamus and pituitary gland. The ventricular system that provides the CSF is divided into four cavities called ventricles, which are connected by a series of holes referred to as foramen, and tubes. Two ventricles enclosed in the cerebral hemispheres are called the lateral ventricles (first and second). They communicate with the third ventricle. The third ventricle is in the center of the brain, and its walls are made up of the thalamus and hypothalamus. The third ventricle connects with the fourth ventricle through a long tube.

\section{BRAIN TUMORS}

A brain tumor is an intracranial mass produced by an uncontrolled growth of cells either normally found in the brain such as neurons, lymphatic tissue, glial cells, blood vessels, pituitary and pineal gland, skull, or spread from cancers primarily located in other organs [2].

Brain tumors are classified based on the type of tissue involved, the location of the tumor, whether it is benign or malignant, and other considerations. Primary (true) brain tumors are the tumors that originated in the brain and are named for the cell types from which they originated. They can be benign (non cancerous), meaning that they do not spread elsewhere or invade surrounding tissues. They can also be malignant and invasive (spreading to neighboring area). Secondary or metastasis brain tumors take their origin from tumor cells which spread to the brain from another location in the body. Most often cancers that spread to the brain to cause secondary brain tumors originate in the lumy, breast, kidney or from melanomas in the skin.

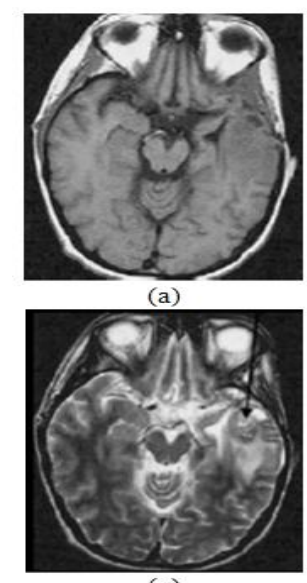

(c)

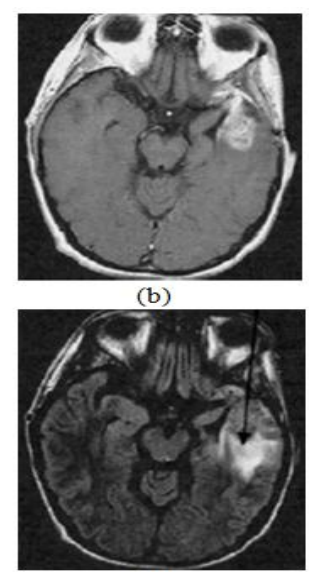

(d)
Fig. 2 MRI of brain (a) T1-weighted image without contrast enhancement (b) T1-weighted image with contrast enhancement (c) T2-weighted image (d) FLAIR image 


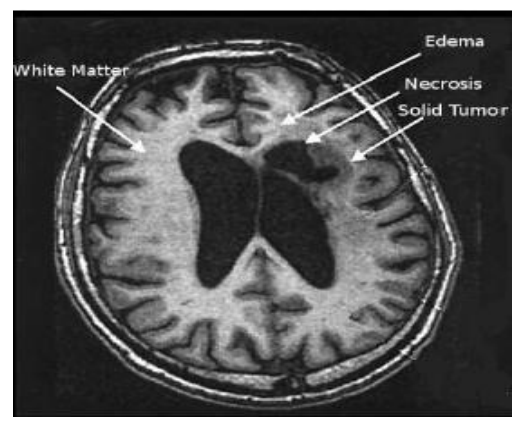

Fig. 3 One axial slice of a MR image of the brain showing tumor areas

Each primary brain tumor, in addition to the solid portion of the tumor, may have other associated parts such as edema and necrosis as in Fig. 2 and 3. Edema is one of the most important factors leading to mortality associated with brain tumors. By definition, brain edema is an increase in brain volume resulting from increased sodium and water content and results from local disruption of the Blood Brain Barrier (BBB). Edema appears around the tumor mainly in white matter regions. Tumor associated edema is visible in MRI, as either hypo-intense (darker than brain tissue) or rarely iso-intense (same intensity as brain tissue) in T1-weighted scans, or hyper-intense (brighter than brain tissue) in T2weighted and FLAIR MRI (Fig. 3). Necrosis is composed of dead cells in the middle of the brain tumor and is seen hypointense in T1-weighted images (Fig. 3). A brain tumor may also infiltrate the surrounding tissues or deform the surrounding structures [4].

\section{CLASSIFICATION OF BRAIN TUMORS}

The classification of primary brain tumors is usually based on the tissue of origin, and occasionally on tumor location. The degree of tumor malignancy is determined by the tumor's histopathologic features. Because of the substantial variety and unusual biology of brain tumors, it has been extremely difficult to develop a widely accepted histological classification system.

The earliest brain tumor classifications were provided by Bailey and Cushing in 1926. Their classification scheme proposed 14 brain tumor types, directed important attention to the process of cell differentiation, and dominated views of gliomas until 1949 when a new system was introduced by Kernohan and Sayre. Kernohan and Sayre made the important realization that different histopathologic appearances may not represent separate tumor types but rather di_erent degrees of differentiation of one tumor type. They classified tumors into five subtypes: astrocytoma, oligodendroglioma, ependymoma, gangliocytoma, and medulloblastoma and very importantly added a four-level grading system for astrocytomas [1]. The grading system was based on increasing malignancy and decreasing differentiation with increasing tumor grade. The addition of a grading system was a very important advance in classifying brain tumors, and provided information not only regarding tumors' biologic behavior but also information that could be used to guide treatment decisions.

\section{BRAIN TUMOR SEGMENTATION}

Despite numerous efforts and promising results in the medical imaging community, accurate and reproducible segmentation and characterization of abnormalities are still a challenging and difficult task because of the variety of the possible shapes, locations and image intensities of various types of tumors. Some of them may also deform the surrounding structures or may be associated to edema or necrosis that changes the image intensity around the tumor. As we surveyed in the previous chapter, existing methods leave significant room for increased automation, applicability and accuracy. Most of them are usually dedicated to full-enhanced tumors or specific types of tumors, and do not extent easily to more general types [5].

The automated brain tumor segmentation method that we have developed consists of two main components: preprocessing and segmentation. The inputs of this system are two different modalities of MR images: CE-T1w and FLAIR that we believe are sufficient for brain tumor segmentation [2]. In the segmentation preprocessing section, operations such as: reduction of intensity inhomogeneity and inter-slice intensity variation of images, spatial registration (alignment) of the input images, segmentation of the brain, computation of the approximate symmetry plane and histogram analysis based on symmetry plane are performed.

\section{PREPROCESSING}

In the real MRI data there are some problems that have to be first solved before any segmentation operation. Therefore we first try to reduce the intensity inhomogeneity and interslice intensity variations, two main problems of MRI data, in the input images. Our system uses two different modalities of MRI, usually not spatially aligned and often having different resolutions. Hence it is required to add a registration and interpolation step. The brain is then segmented by a combination of histogram analysis, morphological operations and symmetry analysis. In this step, we compute the approximate symmetry plane that will be used in the segmentation and sometimes to correct the brain segmentation result. Finally we analyze the histograms of the right and left hemispheres to detect the pathological hemisphere and the type of tumor.

\section{A. Image Preprocessing}

Two main problems of MR images are intensity in homogeneity, or bias field and interslice intensity variation which are caused by the limitations of the current MRI equipments (the main factors are RF excitation field inhomogeneity, non-uniform reception coil sensitivity, eddy currents driven by field gradients, RF penetration and standing wave effects). In today's MR images, the bias field 
is not always visible to the human observer, but it causes significant tissue misclassification problems when intensitybased segmentation is used [7]. Therefore, it is required to correct intensity inhomogeneity in the image volume.

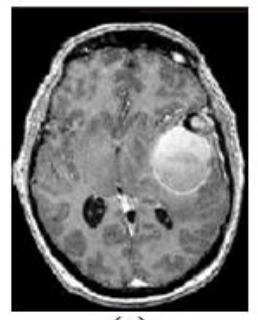

(a)

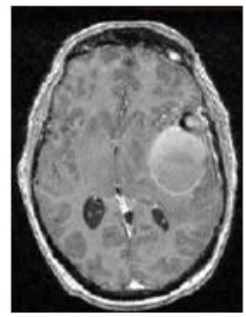

(b)

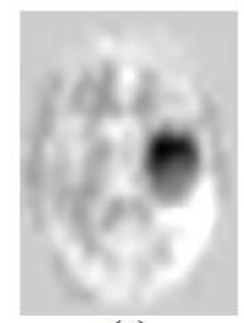

(c)
Fig. 4 Bias field correction (a) An axial slice of the original image (b) Same bias field corrected slice (c) Applied bias field

An automatic method based on entropy minimization is used (as seen in Figure 4). In addition to a smoothly varying field inhomogeneity, two-dimensional multislice sequence MR images, which are acquired in an interleaved way, are typically also corrupted with a slice by slice constant intensity offset. This is usually due to gradient eddy currents and crosstalk between slices. Hence, it is required to normalize interslice intensity to have a correct 3D segmentation. Here a method based on scale-space analysis of histogram is used [9].

\section{B. Image Registration}

Image registration is the operation of aligning images in order to relate corresponding features. For most kinds of image processing on two or more images, it is required that the images are aligned, so that one voxel position represents the same anatomical position in all images [10]. This step allows the use of modalities that are not in perfect alignment. An image registration program has typically four modules: the transformation model, feature extraction, similarity measure, and an optimization method. In our system, the CE-T1w image is used as reference or target image (R) and the FLAIR image as test or source image (T).

Several transformation models can be used to transform the test image $\mathrm{T}$, such as rigid, affine, projection and curved transformations. Here, the registration concerns 3D head images from the same person, which makes it reasonable to assume that the head will not be deformed, and thus can be considered a rigid body. Hence, the rigid transformation model (rotation and translation) is therefore sufficient for our purpose. By using a rigid transformation, we are assuming that the two images can be aligned using a parameterization with 6 degrees of freedom. Here we restrict ourselves to methods that use directly the intensity images as features, thus avoiding the preliminary extraction of corresponding features in the two images.

\section{Brain Segmentation}

The next step of preprocessing consists of brain segmentation. Several methods have been proposed to perform this operation and some of them are available in softwares such as Brain-Visa, FSL and Brain suite. Unfortunately most of them fail in the case of the presence of a tumor in the brain, especially if located on the border of the brain (Figure 5). To solve this problem, we propose to perform a symmetry analysis, based on the assumption that tumors are generally not symmetrically placed in both hemispheres, while the whole brain is approximately symmetrical.

First we segment the brain using histogram analysis and morphological operations. This leads to a partial segmentation, where a part corresponding to the tumor may be missing. The algorithm is applied on the gray level image of the head to compute the approximate symmetry plane, because the segmented brain is not symmetric. The computed symmetry planes of the head and of the segmented brain in normal cases are approximately equal and this approximation is acceptable in pathological cases for tumor detection purpose.
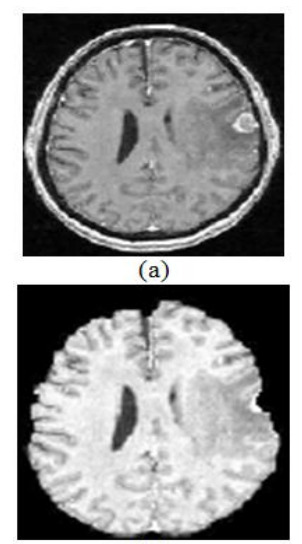

(c)
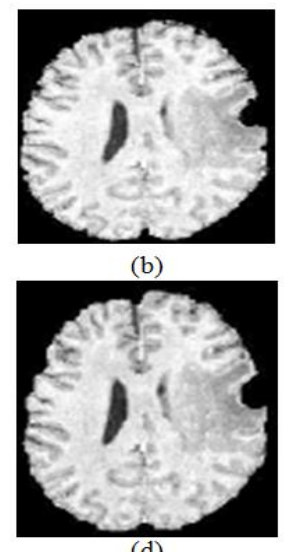

(d)
Fig. 5 Pathological brain segmentation using existing methods

(a) One slice of the original image on two examples (b) Segmented brain by histogram analysis and morphological operations using Brain Visa (c) Segmented brain by BET using FSL

(d) Segmented brain by BSE using Brain suite

\section{Structure Segmentation}

The proposed method for internal brain structures segmentation, such as for tumors, has two phases: initialization and refinement. In other words, we first segment the brain tissues (consequently the internal structures of the brain) and since this segmentation for internal brain structures is not fine enough, we then refine them one by one using prior information. To perform these two phases, the segmentation procedure consists of the following steps [6]:

1. Global segmentation of the brain,

2. Retrieving spatial relations,

3. Selecting the valid spatial relations,

4. Fuzzification and fusion of relations and providing the ROI,

5. Searching the initial segmentation of structure, 
6. Refining the initial segmentation,

7. Repeating from step 2 for other structures.

For global segmentation of the brain to segment the brain tissues and its structures, we use two methods, the first one is the MPFCM method and the second one is the multiphase level sets.

\section{PERFORMANCE MEASURES}

Characterizing the performance of image segmentation methods is a challenge in image analysis. An important difficulty we have to face in developing segmentation methods is the lack of a gold standard for their evaluation. Accuracy of a segmentation technique refers to the degree to which the segmentation results agree with the true segmentation. Although physical or digital phantoms can provide a level of known "ground truth", they are still unable to reproduce the full range of imaging characteristics and normal and abnormal anatomical variability observed in clinical data.

Manual segmentation of desired objects by domain experts can be considered as an acceptable approach (it still suffers from inter-expert and intra-expert variability). The result of an automated method is then compared to the manually segmented object by an expert or a group of experts, and if the algorithm generates segmentations sufficiently similar to the ones provided by the experts, it is accepted. A number of metrics have been proposed to measure the similarity between the segmentations, including volume measures and surface measures.

\section{EXPERIMENTAL RESULTS}

In order to implement this 3D brain tumors and internal brain structures segmentation in MR images efficiently, VB.NET program is used. This program could speed up the development of this system because it has facilities to draw forms and to add library easily [6].

The proposed method was applied to 10 clinical MRI datasets of various origins and types. We illustrate the results on four cases, for which manual segmentation of several structures was available, and which exhibit tumors with different shapes, locations, sizes, intensities and contrasts. Evaluation of the segmentation results was performed through quantitative comparisons with manual segmentations, using volume and surface measures. Segmentation results are quantitative evaluations are high accuracy. The voxel size is typically $1 \times 1 \times 1.3 \mathrm{~mm} 3$, so that the average error is less than one voxel. The Hausdorff distance represents the error for the worst point, which explains its higher values. Although the segmented structures are relatively small (about $4000 \mathrm{~m} 3$ ), the volume metrics shows good results. For the similarity index measures, values above $70 \%$ are satisfactory. The results show that the segmentation of caudate nuclei is better than thalamus due to their well defined borders. The comparison of the results obtained using the initial segmentation of MPFCM and multiphase level sets illustrates that there is not a large difference between them. But the MPFCM method is faster than the multiphase level sets method.

\section{CONCLUSION}

In this paper, we deal with $3 \mathrm{D}$ MR images in order to segment brain tumors and internal brain structures for the applications such as treatment and follow-up, surgery, individual modeling, etc. We first review the discussed topics and the contributions and following this, we discuss possible future directions. We proposed a new method for segmentation of pathological brain structures. This method combines prior information of structures and image information (region and edge) for segmentation. To represent the prior information we used ontological engineering tools. We also proposed a simple ontology for a specific classification of tumors and it can be extended for other classification of tumors (such as tumor grading). Brain tumor segmentation method uses contrast enhanced T1weighted and FLAIR images for segmentation and it consists of two steps: preprocessing and segmentation. In the preprocessing step, in addition to use of the classical methods for reducing the noise and inhomogeneity and registration, we proposed a new adapted method for correct and robust brain segmentation. The brain is segmented by a combination of histogram analysis, morphological operations and symmetry analysis. A new symmetry-based histogram analysis was proposed that is able to detect automatically the tumor type and the pathological hemisphere.

The segmentation of the pathological brain structures is a difficult task due to the different effects of the different tumors. Using prior information such as an atlas or adapting it to guide the segmentation is also difficult because of these different effects. We proposed a new method that in addition to region and edge information uses a type of prior information which is more consistent in pathological cases. The spatial relations between structures are the prior information used in this method. Here we deal with three main problems: explicit representation of spatial relations for each structure, adaptation of spatial relations for pathological cases and segmentation method and volume considered an important parameter such as, storage, transmission, visualization, and quantitative analysis.

\section{REFERENCES}

[1] M. E. Algorri, and F. Flores-Mangas, "Classification of anatomical structures in MR brain images using fuzzy parameters" IEEE Transactions on Biomedical Engineering, Vol. 51, No. 9 , pp. 1599$1608,2004$.

[2] W. Dou, S. Ruan, Y. Chen, D. Bloyet, and J. M. Constans, "A framework of fuzzy information fusion for segmentation of brain tumor tissues on MR images", Image and Vision Computing, Vol. 25, pp. 164-171, 2007.

[3] L. M. Fletcher- L. O. HeathHall, D. B. Goldgof, and F. Murtagh, "Automatic segmentation of non-enhancing brain tumor in magnetic 
resonance images", Artificial Intelligence in Medicine, Vol. 21, pp. 43-63, 2001.

[4] D.T. Gering, "Recognizing Deviations from Normalcy for Brain Tumor Segmentation", $\mathrm{PhD}$ thesis, Massachusetts Institute of Technology, 2003.

[5] N. Hata,Y. Muragaki, T. Inomata, T. Maruyama, H. Iseki, T. Hori, and T. Dohi, "Intera-operative tumor segmentation and Vol. measurement in MRI guided glioma surgery for tumor resection rate control", Academic Radiology, Vol. 12, pp. 116-122, 2005.

[6] S. Hu, and D. L. Collins, "Joint level-set shape modeling and appearance modeling for brain structure segmentation", Neuro Image, Vol. 36, pp. 672-683, 2007.
[7] K. M. Iftekharuddin, W. Jia, and R. Marsh, "Fractal analysis of tumor in brain MR images", Machine Vision and Applications, Vol. 13, pp. 352-362, 2003.

[8] H. Khotanlou, O. Colliot, and I. Bloch, "Automatic Brain Tumor Segmentation using Symmetry Analysis and Deformable Models", In International Conference on Advances in Pattern Recognition, pp. 198-202, Kolkata, India, 2007.

[9] A. Lefohn, J. Cates, and R. Whitaker, "Interactive, GPU-based level sets for 3D brain tumor segmentation", Technical report, University of Utah, 2003.

[10] J. Maintz, and M. Viergever, "A survey of medical image registration”, Medical Image Analysis, Vol. 2, No. 1, pp. 1-3, 1998. 